Ambiances

anbiances Environnement sensible, architecture et espace urbain

$2 \mid 2016$

Ambiance et histoire de l'architecture : l'expérience et l'imaginaire sensibles de l'environnement construit

\title{
Les ambiances dans les récits de visite : une source pour l'étude de la réception de l'architecture
}

L'exemple du nouveau Musée du Luxembourg à Paris en 1886

Atmosphere in visitor reviews : a source for the reception theory of architecture.

The "new " Luxembourg Museum in 1886 : a case study

Julien Bastoen

(2) OpenEdition

Journals

Édition électronique

URL : http://journals.openedition.org/ambiances/789

DOI : 10.4000/ambiances.789

ISSN : 2266-839X

Éditeur :

Direction Générale des Patrimoines - DAPA - MCC, UMR 1563 - Ambiances Architectures Urbanités $(\mathrm{AAU})$

Référence électronique

Julien Bastoen, «Les ambiances dans les récits de visite : une source pour l'étude de la réception de l'architecture », Ambiances [En ligne], 2 | 2016, mis en ligne le 07 novembre 2016, consulté le 30 avril 2019. URL : http://journals.openedition.org/ambiances/789; DOI : 10.4000/ambiances.789

Ce document a été généré automatiquement le 30 avril 2019

\section{$($ C) $(1) \Theta$}

Ambiances is licensed under a Creative Commons Attribution-NonCommercial-NoDerivatives 4.0 International License. 


\title{
Les ambiances dans les récits de visite : une source pour l'étude de la réception de l'architecture
}

\author{
L'exemple du nouveau Musée du Luxembourg à Paris en 1886
}

Atmosphere in visitor reviews : a source for the reception theory of architecture. The " new » Luxembourg Museum in 1886: a case study

Julien Bastoen

1 Depuis une vingtaine d'années, plusieurs études ont ouvert la voie à une histoire de la muséographie du point de vue des ambiances aux XIX ${ }^{\mathrm{e}}$ (Georgel, 1994) et XX ${ }^{\mathrm{e}}$ siècles (Simonnot, 2011a, 2011b, 2012), une période cruciale qui coïncide non seulement avec la professionnalisation et la structuration des métiers d'architecte et de conservateur, mais aussi avec l'affirmation de la muséographie comme science autonome.

2 Les composantes des ambiances recouvrent en effet au moins partiellement les paramètres à prendre en compte pendant la phase de programmation d'un musée, dans le cadre d'une réflexion sur la muséographie et la conservation préventive des collections : "L'ambiance, même si sa traduction est technique - lumière, matières, transparence, densité, flux, fréquentation, échelle, volume... la liste est infinie -, est avant tout conceptuelle, elle se situe à l'amont » (Grange et Petit, 2007).

3 Toute étude rétrospective se heurte néanmoins à des apories méthodologiques, qui sont notamment l'altération, voire la disparition des bâtiments, et la difficulté de trouver des archives ou même de simples descriptions, graphiques ou écrites, permettant de documenter la construction, l'aménagement et la réception des musées. Il est possible de contourner une partie du problème en considérant les récits de visite comme des sources exploitables pour comprendre l'expérience de la visite comme fait de société (Poulot, 1983, 1994), mais aussi pour évaluer l'influence des ambiances sur cette expérience.

4 Considérant que les représentations d'ambiances ne sont pas uniquement graphiques, mais aussi langagières, écrites ou orales (Drozd, 2008a, 2011), et qu'elles ne sont pas 
l'exclusivité des architectes, nous postulons que les récits de visite, qui accordent une large place à la description d'objets, de lieux et d'ambiances, constituent une source incontournable pour qui souhaite restituer la réception d'un lieu, en particulier d'un musée, en même temps qu'ils permettent d'appréhender les ambiances à partir de l'usager et non pas du concepteur.

Comment restituer, par exemple, la réception du premier musée d'art construit et aménagé à Paris au XIX ${ }^{e}$ siècle? Alors que les autres capitales européennes et les chefslieux de départements français se dotent, au XIX siècle, de musées monumentaux (Hamon, 2004), il faut attendre 1886 pour voir l'ouverture, à Paris, du « nouveau » Musée du Luxembourg, alors vitrine nationale pour l'art contemporain (Lacambre, 2003), sur le site où il existe encore aujourd'hui, avec des missions et un statut différents. Ce pionnier des musées d'art contemporain, au point que son nom en est devenu le synonyme au XIX ${ }^{\mathrm{e}}$ siècle, fut installé en 1818 au palais du Luxembourg, qu'il partagea principalement avec le Sénat, et temporairement avec la Préfecture de la Seine (1871-1879). Le Sénat, rétabli en 1875 avec des missions élargies, réclama en 1879 la récupération des espaces occupés par le musée pour s'agrandir. Après cinq ans de négociations avec l'administration des BeauxArts, tutelle du musée, le Sénat proposa de financer le relogement temporaire des collections dans un bâtiment proche du palais, l'orangerie du Luxembourg, une serre orientée plein sud construite en 1842, qui sera transformée et agrandie entre 1884 et 1886 selon un projet dessiné par les architectes Charles Gondoin et Georges Scellier de Gisors.

6 La réouverture du musée dans ses nouveaux locaux constitue un événement architectural autant qu'artistique. Les attentes du champ artistique - à commencer par les artistes vivants eux-mêmes, premiers bénéficiaires de cette instance de consécration - sont considérables, dans la mesure où l'on attend de ce musée national, qui abrite principalement des peintures et des sculptures, qu'il soit exemplaire par son architecture et sa muséographie, et qu'il soit digne de l'attractivité et du rayonnement internationaux de la capitale française. Or, cet événement n'a jamais été analysé de manière objective ; un seul article, écrit par un conservateur du Musée du Luxembourg cinquante ans après (Ladoué, 1936), représente une tentative incomplète et nécessairement partiale de rendre compte de cette nouveauté architecturale. Le bâtiment lui-même, largement altéré depuis 1886, à l'intérieur comme à l'extérieur, ne peut, en tant que tel, être considéré comme une source de première main. En outre, la plupart des sources visuelles de l'extérieur et de l'intérieur du musée étant postérieures, l'historien ne peut les utiliser pour appréhender la réception immédiate du nouveau musée. Les récits de visite publiés autour du moment de l'inauguration s'imposent donc comme le principal corpus exploitable, à condition d'éliminer de ce corpus les témoignages qui ne contiennent que des données factuelles ou n'évoquent à aucun moment la configuration et la perception sensible des lieux. Nous nous appuierons sur une sélection de récits signés ou anonymes, extraits d'un large choix de périodiques - presse généraliste, presse illustrée, presse spécialisée du champ artistique -, caractéristiques de l'échiquier politique de l'époque : la diversité des genres et des tendances politiques des supports permet d'établir un échantillon représentatif de la réception critique externe. Il s'agira d'être attentif aux champs lexicaux, aux procédés d'écriture, aux convergences ou divergences d'impressions, mais également aux connotations et à l'instrumentalisation de ces descriptions pour faire valoir ou, au contraire, décrédibiliser la compétence des acteurs de l'opération. Nous mettrons ces témoignages sur les ambiances à l'épreuve des connaissances issues des archives sur l'amont et l'aval de l'opération, afin de valider 
l'hypothèse d'un hiatus entre la vision des architectes et sa matérialisation, révélateur de dysfonctionnements et de négligences, de conflits de compétence ou d'autorité, pendant le processus de projet.

\section{Un monument indigne du rayonnement culturel de Paris?}

\section{Un bâtiment hybride}

7 La réception du «nouveau musée » du Luxembourg ne se concentre pas exclusivement autour du moment de la visite réservée à la presse, le 30 mars 1886, et de l'inauguration officielle, célébrée le $1^{\mathrm{er}}$ avril sous la houlette du Président de la République Jules Grévy, réélu trois mois plus tôt. Et pour cause : une grève des mineurs à Decazeville et une flambée insurrectionnelle en Belgique monopolisent l'attention des principaux quotidiens, reléguant la nouvelle de la réouverture du musée au mieux sur une colonne, au pire, sous la forme d'un entrefilet. L'inauguration de la rétrospective posthume de l'œuvre du peintre académique Paul Baudry, disparu quelques mois auparavant, a même tendance à éclipser celle du nouveau Musée du Luxembourg. Il faut donc considérer un laps de temps plus long, entre l'automne 1885 et l'été 1886, pour trouver suffisamment de sources exploitables. Parmi les extraits de récits de visite sélectionnés dans la presse, signés ou anonymes, la question du parti pris architectural, c'est-à-dire l'idée directrice du projet, est sans doute celle qui divise le plus les commentateurs. De l'enthousiasme le plus explicite aux attaques les plus virulentes, la diversité des prises de position confirme au moins l'intérêt porté par les journalistes à cet objet architectural inédit dans le paysage parisien. Le critique d'art Charles Clément annonce qu'« on étudiera ce nouveau Musée, avec d'autant plus d'intérêt qu'il est établi dans des conditions toutes nouvelles dans notre pays " (Clément, 1886), rejoignant le discours officiel d'Étienne Arago, le conservateur en chef du musée, qui observe qu'il s'agit « du premier monument qui ait été construit à Paris pour un musée de peinture et de sculpture " (Arago, 1886, p. XXI), alors que les musées de province, parfois construits ad hoc, commencent à pulluler depuis le Second Empire (Sherman, 1989; Hamon, 2004; Bertinet, 2015). L'extension de l'orangerie Férou apparaît en effet, après la construction de la galerie de minéralogie au Jardin des Plantes, comme le second bâtiment parisien spécifiquement construit pour remplir une fonction exclusivement muséographique, et le premier à vocation artistique : le Palais des études de l'Ecole des Beaux-Arts n'abrite pas qu'un musée; le chantier du musée Galliera, entamé en 1879, n'est achevé qu'en 1894, et celui du musée Guimet ne débute qu'en 1886.

Le Musée du Luxembourg que les journalistes, puis les visiteurs, découvrent à partir du 30 mars 1886, est donc un édifice hybride, associant construction neuve et restructurationextension d'une serre initialement conçue pour concentrer la chaleur en hiver comme en été. La longueur de l'ancienne orangerie construite par Alphonse de Gisors en 1839 dans le périmètre du jardin du Luxembourg, près de la rue de Vaugirard, a été portée de sept à neuf travées, tout en conservant son homogénéité stylistique, néo-renaissance, associant brique et pierre. Les sept travées du bâtiment d'origine ont été compartimentées en neuf salles de peintures, les deux nouvelles travées accueillant un salon carré. L'ensemble de la toiture d'origine a été déposé et remplacé par des doubles combles vitrés sur toute la longueur du bâtiment. 
Bien que les orangeries ne se prêtent pas, a priori, à un usage muséographique, dans la mesure où la température peut y atteindre 50 degrés, la tradition de leur reconversion remonte à la fin du XVIII e siècle. Quelques années avant la transformation de l'orangerie du Luxembourg, la collection Herzog Anton-Ulrich est installée, entre 1871 et 1877, dans l'orangerie de Kassel apprêtée par l'architecte Heinrich von Dehn-Rotfelser; en 1876, la pinacothèque de Francfort déménage dans une orangerie remaniée par l'architecte Oskar Sommer, qui devait récidiver sept ans après à Brunswick. Enfin, pratiquement au même moment, à partir de 1878, l'orangerie des Tuileries accueillit périodiquement des expositions en tout genre, après avoir servi accessoirement de dépôt des marbres.

Un bâtiment bas a été greffé aux deux nouvelles travées de l'orangerie, en retour d'équerre ; éclairé, lui aussi, par un double comble vitré, il accueille la sculpture dans un volume unitaire. Dans le prolongement de cette galerie de sculpture, un pavillon en brique et pierre, au néo-classicisme discret matérialisé par l'usage de pilastres jumelés surmontés d'un fronton au tympan sculpté, abrite le vestibule d'entrée du musée, les bureaux du conservateur en chef et du gardien en chef ainsi que les toilettes et la chaufferie. On accède à ce pavillon par un large escalier, précédé d'une cour ouvrant sur la rue de Vaugirard, face à la rue Férou. Le musée bénéficie d'un accès indépendant de celui du jardin du Luxembourg, le plus ancien jardin de Paris ouvert au public, dans lequel il se situe néanmoins.

Illustration 1 : Vue cavalière du nouveau Musée du Luxembourg vers le sud-est.

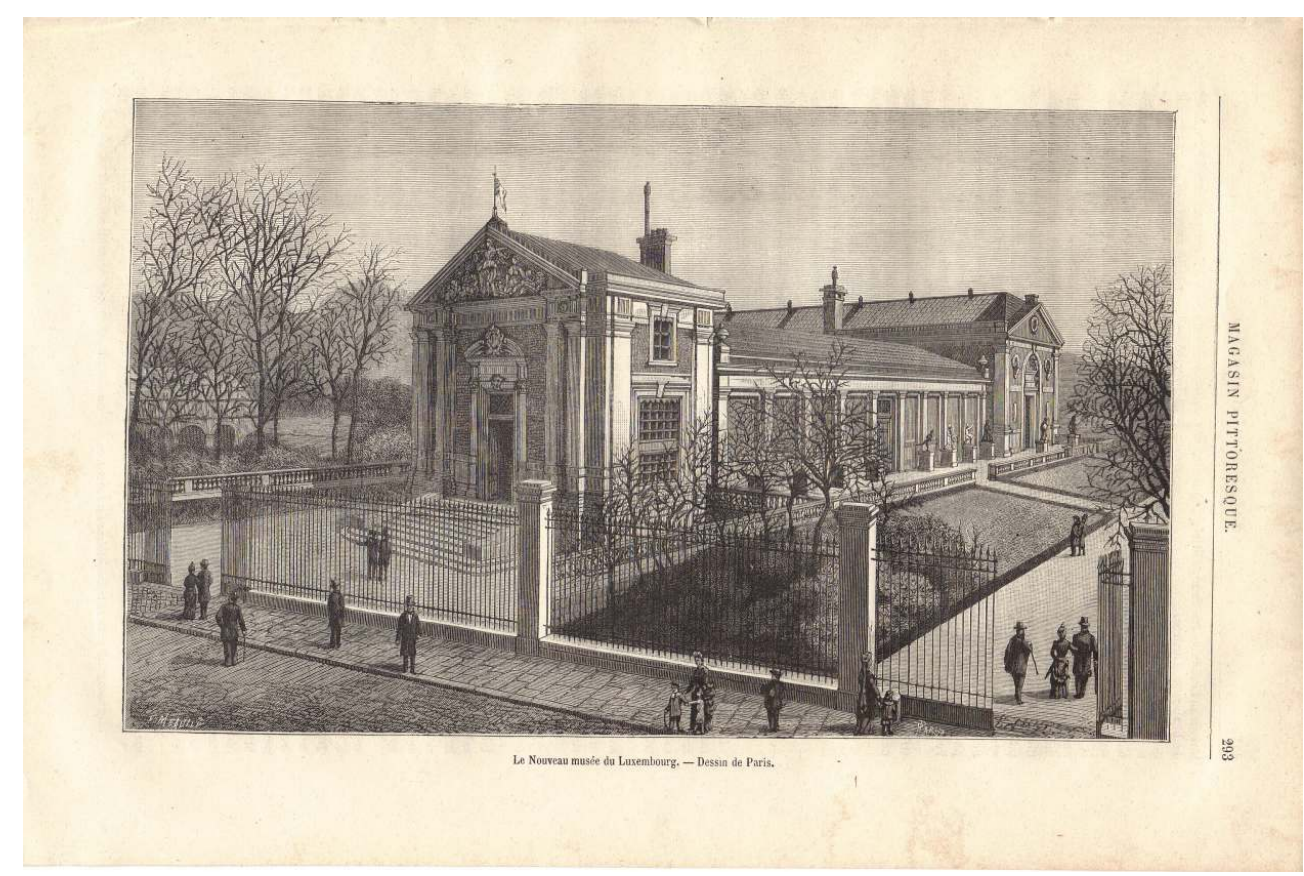

Source: Paris, «Le Nouveau musée du Luxembourg », Le Magasin pittoresque, 1888, p. 293. Copyright: collection de l'auteur.

\section{Une exemplarité architecturale contestée}

11 L'enjeu consiste alors, pour la presse, à déterminer si ce musée est digne de s'ériger en modèle, et d'abriter les collections nationales d'art contemporain, dans un contexte de plus en plus concurrentiel à l'échelle européenne (Pomian, 1994): Londres, Vienne, 
Bruxelles, possèdent des salles réservées aux artistes contemporains dans leurs principaux musées d'art; la Pinacothèque nouvelle à Munich et la Galerie nationale à Berlin, achevées respectivement en 1853 et 1876, se sont imposées comme des paradigmes architecturaux et muséographiques pour l'art contemporain dans le reste de l'Europe ; Rome ouvre sa Galerie nationale d'art moderne en 1883 dans un Palais des Expositions flambant neuf (Lorente, 2009).

Le mérite des architectes, compte tenu de la complexité de l'opération, est reconnu aussi bien dans la presse généraliste que dans les revues spécialisées : Charles Clément, dans le conservateur Journal des débats, considère que «ce programme de construction, parfaitement adaptée à sa destination, exécutée rapidement et dans des conditions nécessaires d'économie [...], dans son genre, est un modèle. Le monument est très simple, mais élégant, de bon goût, clair et gai » (Clément, 1886); deux quotidiens républicains, Le Temps et Le XIXe siècle, célèbrent respectivement «une demeure bien située, bien distribuée et largement ouverte à la lumière " (Anonyme, 1886a), "aux bonnes proportions et à la belle ordonnance de l'ensemble » (Roger, 1886). Du côté de la presse spécialisée, Marcel Daly, co-directeur de la Semaine des Constructeurs, souligne l'élégance du bâtiment symbolisée par sa porte principale qu'il choisit pour illustrer son article (Daly, 1886) ; l'architecte, critique et historien de l'architecture Maurice du Seigneur, reconnaît dans le magazine La Construction moderne, fondé en 1885 par l'ingénieur Paul Planat, que l'architecte a «employé avec un goût parfait l'union de la brique et de la pierre qui, autrement mises en œuvre, auraient pu faire ressembler ce sanctuaire des arts à des écuries ou à des communs » (Du Seigneur, 1886).

13 L'exemplarité architecturale du « nouveau Luxembourg » semble indiscutable, si l'on s'en tient au fait que l'inauguration du bâtiment coïncide avec la consécration de l'un des auteurs du projet : Georges Scellier de Gisors, assistant de Charles Gondoin, l'architecte en chef du palais du Luxembourg et du Sénat, et auteur de la plupart des esquisses du projet, bien qu'il n'ait pas eu le droit de les signer. Sur proposition du président du Sénat, validée par le ministère de l'Instruction publique et des Beaux-Arts, Scellier de Gisors reçoit sa médaille de chevalier de la Légion d'honneur des mains du président de la République, Jules Grévy, le jour de l'inauguration du nouveau musée.

Cette convergence apparente d'impressions positives, qui pourrait convaincre le lecteur que «le Musée du Luxembourg est digne de recevoir les chefs-d'œuvre de l'École française moderne » (Katow, 1886), est toutefois remise en question par les descriptions bien moins consensuelles publiées dans la presse. Les caractéristiques considérées comme des qualités par les uns deviennent, pour d'autres, les principaux défauts. La plupart des critiques se concentrent sur l'inadéquation du bâtiment à sa mission et à son rayonnement international, et n'hésitent pas à user de périphrases ou d'analogies péjoratives pour dévaloriser le bâtiment et décrédibiliser l'architecte, et à travers eux, la politique artistique de l'Etat: le critique d'art Edmond Bazire, alias Edmond Jacques, excommunard, défenseur de Manet, Pissarro et Rodin, écrit dans L'Intransigeant, un quotidien socialiste, que « de ce vieux bâtiment plat et bas, on a tiré tout le parti possible, c'est-à-dire un mauvais parti, et, à première vue, le passant est offusqué par cette maçonnerie sans élégance. Il se croirait volontiers devant une grange, sans fenêtres, sans ouvertures, sans ornements» (Jacques, 1886). L'ancien directeur des Beaux-Arts, Paul Mantz, dont on pourrait attendre une position plus consensuelle, qualifie toutefois le nouveau musée de «petite maison » et de «minimum de pinacothèque » dans Le Temps, quotidien républicain conservateur (Mantz, 1886). Dans le même esprit, le quotidien 
républicain Le Rappel, laisse entendre que la médiocrité du résultat est logique, eu égard au manque d'ambition du programme (Anonyme, 1886b).

La première partie de l'analyse des impressions de visite, uniquement centrée sur l'appréhension globale du bâtiment, permet déjà de mettre en évidence des divergences de points de vue qui transcendent l'orientation politique des journaux et le statut des locuteurs. L'analyse des récits de visite de l'intérieur du musée parus dans la presse permet-elle de confirmer ce premier constat?

\section{L'ambiance intérieure du nouveau musée : des impressions contradictoires}

\section{Accessibilité, circulation, confort}

L'accessibilité est sans conteste la seule caractéristique du nouveau musée à faire l'objet d'une reconnaissance unanime dans la presse, et ce, avant même l'inauguration (Anonyme, 1885b ; Fournel, 1886). En effet, le spectre de l'ancien escalier « en casse-cou » (Du Seigneur, 1886 ; Roger, 1886 ; Ponsonailhe, 1886), qui reliait la section de sculpture au rez-de-chaussée du palais du Luxembourg à la section de peinture au premier étage, hante encore la mémoire collective ; l'ancien directeur général des Beaux-Arts confirme la dangerosité de cet accès en le comparant à « une misérable échelle, digne de tous les mépris. Du matin jusqu'au soir, on y entendait le bruit des chutes, et la mère en défendait l'ascension à ses enfants» (Mantz, 1886). Le procédé de la comparaison, utilisé massivement par les commentateurs, tourne donc nécessairement à l'avantage du nouveau musée, et autorise le conservateur à écrire, dans l'introduction au nouveau catalogue, que « toutes les salles destinées tant à la peinture qu'à la sculpture [...] offrent aux visiteurs une circulation facile » (Arago, 1886).

Le constat unanime de l'étroitesse et du «manque de recul » (Mantz, 1886; Roger, 1886) qui caractérisait les salles anciennement occupées par le Musée du Luxembourg incite certains commentateurs à estimer, par contraste, que l'ampleur des espaces du nouveau musée, en particulier dans la section de peintures, constitue un relatif progrès (Clément, 1886 ; Jacques, 1886). De même, chacun se plaît à rappeler qu'auparavant « la sculpture était entassée dans le plus impossible des rez-de-chaussée " (Anonyme, 1885a), "une sorte de cave étroite et humide» (Du Seigneur, 1886), «dans des couloirs tellement resserrés que l'étude y devenait impossible, car l'œil n'apercevait plus que la confusion des lignes et le conflit des silhouettes» (Mantz, 1886). La nouvelle galerie de sculpture suscite néanmoins des impressions discordantes sur un point essentiel : les proportions de cet espace, jugées suffisantes (Mantz, 1886; Clément, 1886; Fournel, 1886) ou insuffisantes (Anonyme, 1886e ; Jacques, 1886) pour pouvoir circuler autour des œuvres et les contempler dans les meilleures conditions.

Par ailleurs, quelques commentateurs n'hésitent pas, avant et après l'inauguration, à souligner le fait que la nouvelle version du Musée du Luxembourg, par son indifférence au confort de visite et de contemplation, accuse déjà du retard par rapport à ses concurrents étrangers. En effet, la Galerie nationale et le Musée d'art et d'industrie de Berlin sont équipés de buvettes, quand l'Ermitage de Saint-Pétersbourg propose des sièges mobiles à ses visiteurs; au Musée du Belvédère, à Vienne, les tableaux sont 
accrochés sur des panneaux mobiles manipulables à la demande des visiteurs (Anonyme, 1885b).

\section{Lumière, couleurs et matériaux}

19 Avec la question de la circulation et du confort des visiteurs, celle du rapport entre l'éclairage et le revêtement des sols et murs cristallise également des opinions contradictoires. Les commentateurs s'accordent, une fois encore, sur l'inadaptation des anciennes salles du palais du Luxembourg à l'exposition de tableaux :

La plupart des ouvrages étaient placés dans des couloirs obscurs, lieux profonds et voisins de l'empire des morts, où il était impossible de distinguer seulement les jaunes des violets et une femme d'un léopard. Cependant d'autres, exposés à une lumière trop crue, reflétaient les rayons du soleil comme des plaques de cuivre. (Anonyme, 1886c)

20 Néanmoins, si les nouvelles salles de peintures, aux murs peints en rouge antique et soulignés de boiseries noires, suivant un modèle répandu à l'époque (Georgel, 1994), sont, pour les uns, "décorées de la manière la plus noble et la mieux entendue » (Clément, 1886), et «offrent un aspect d'une richesse sévère parfaitement en harmonie avec la destination de l'édifice» (Anonyme, 1886a), pour les autres, la teinte choisie pour les murs « est trop rougeoyante " (Mantz, 1886), et devrait être «foncée jusqu'au brun, car elle tue tous les tableaux indistinctement " (Méa, 1886), suivant un principe largement admis dès la seconde moitié du XIX siècle dans la plupart des musées français.

De la même manière, l'éclairage zénithal systématisé dans les salles des peintures inspire des réactions tout aussi contradictoires, alors même que cette solution s'est imposée, dès la fin du XVIII ${ }^{e}$ siècle, comme la meilleure possible pour éclairer la peinture (Georgel, 1994). Pour les uns, ces salles reçoivent une lumière homogène et favorable (Anonyme, 1886c ; Clément, 1886) et produisent une « impression excellente » (Roger, 1886), au point de dépasser en qualité la plupart des salles du Louvre (Anonyme, 1886c). D'autres évoquent prudemment une "lumière loyale et quelque peu intransigeante" (Mantz, 1886), ou dénoncent un "diable d'éclairage» (Jacques, 1886). Edmond Bazire prétend même qu'« il descend de grandes tâches noires sur les figures claires ou de grands coups de soleil sur les figures sombres, et [qu']il est impossible de concevoir l'idée de l'auteur j'allais écrire de la victime » (ibid.).

22 Dans la salle de sculpture, tandis que la tonalité rouge des murs ne fait que reprendre une solution déjà approuvée dans d'autres musées, notamment ceux d'Amiens et Douai, le recours à l'éclairage zénithal sur toute sa surface rompt avec la tradition de l'éclairage latéral haut prôné jusqu'alors. Si Paul de Katow, peintre et critique redouté du Gil Blas, s'enthousiasme pour cette "vaste salle, parfaitement décorée, admirablement éclairée " (Katow, 1886), le dépolissage, responsable d'un éclairage trop cru, voire aveuglant, est jugé insuffisant par un certain nombre de commentateurs (Roger, 1886 ; Clément, 1886). La femme peintre et critique d'art, Sabine Méa, jamais représentée dans les collections du Musée du Luxembourg et particulièrement active dans le débat sur la situation architecturale du musée depuis le début des années 1880, souligne la confusion visuelle engendrée par l'excès de lumière, l'accumulation d'objets et un sol trop clair (Méa, 1886). Si ces impressions divergentes peuvent en partie s'expliquer par des conditions d'ensoleillement variables selon le moment de la visite, il n'en reste pas moins qu'il n'y a 
pas unanimité, ce qui n'est pas sans poser question sur les raisons du choix du dispositif d'éclairage.

\section{Le climat du musée : surchauffe et humidité} visite. Critique d'art favorable aux impressionnistes et symbolistes en particulier, jusquelà peu ou pas représentés dans les collections du Luxembourg, Charles Ponsonailhe pointe principalement la mauvaise conception du dispositif de double peau destiné à atténuer l'effet de serre produit par les larges baies ouvertes sur la façade sud de l'orangerie: "pourquoi ces murs pleins, sans une seule baie permettant l'aération, facilitant l'établissement de courants d'airs?» (Ponsonailhe, 1886). Plusieurs commentateurs s'accordent sur le fait que le dépolissage insuffisant du verre des plafonds des salles de sculpture et de peinture, autre facteur d'augmentation substantielle de la température dans le bâtiment et de dégradation des toiles, pourrait être corrigé par l'installation d'un système de stores. C'est d'ailleurs en réaction à un article de Sabine Méa qui rappelle ironiquement que «le local a changé de destination, et qu'il n'est plus question d'y cultiver des caféiers et des bananiers » (Méa, 1886), qu'un lecteur anonyme plaide pour une prise en considération urgente et sérieuse de ce paramètre :

Dimanche dernier, alors que la température était excellente dans la rue, beaucoup de personnes se trouvaient mal à l'aise dans les galeries. C'était un cri général de tous côtés. Chacun regardait d'un fort mauvais œil ces plafonds de verre dépourvus de toute espèce de toile. (Anonyme, 1886d)

Au problème de la chaleur, s'ajoute celui de l'humidité. Charles Ponsonailhe et Edmond Bazire l'évoquent sur un ton corrosif, dans les deux mois qui suivent l'inauguration. Le premier met ainsi en cause la logique de l'économie maximum, qui a conduit le Sénat à choisir un emplacement par défaut, sans cave et sur un terrain non drainé, inadapté à la construction d'un musée (Ponsonailhe, 1886). Edmond Bazire, lui, compare le nouveau musée à un bagne, analogie déjà utilisée par un chroniqueur, Pierre Véron, à propos des anciens locaux :

La menaçante humidité m'inquiète. Ils [les tableaux] n'étaient condamnés par le Sénat qu'à la proscription. Le sous-secrétaire d'État à l'instruction publique les condamne à mort. Je ne sais par quelle liaison d'idées, le lien de leur relégation me faisait penser à la loi sur les récidivistes. C'est leur Guyane que l'Orangerie. Seulement, au lieu d'y trouver la guillotine sèche, ils y trouveront la guillotine mouillée. (Jacques, 1886)

L'analyse des récits de visite, et plus spécifiquement des descriptions du nouveau musée consacré à l'art français contemporain en 1886, révèle donc des points de vue divergents sur les mêmes composantes d'ambiance. Les contradictions mises en évidence par le croisement des témoignages invitent à relativiser autant la satisfaction de la maitrise d'ouvrage que la fiabilité de certains commentateurs, manifestement consensuels. Si la comparaison des nouveaux espaces occupés par les collections avec les anciens est plutôt à l'avantage des premiers, un certain nombre de journalistes pointent du doigt des problèmes qui, si leurs propos sont de bonne foi, ont dû se répercuter dans les altérations ultérieures du bâtiment et l'évolution de ses usages. Or, on constate, en confrontant les archives administratives et les articles de presse produits dans les mois qui suivent l'ouverture du nouveau musée, qu'un certain nombre de mesures, restrictives et correctives, sont prises par le conservateur en chef, au risque de créer la polémique dans 
le champ artistique: annulation du projet de création d'une section de gravure, ajournement du projet de création d'archives de l'art contemporain, instauration d'un quota d'œuvres par artiste. Ces mesures qui trahissent le fait que le musée, à peine inauguré, est déjà saturé, sont associées à d'autres qui concernent moins la gestion des collections et la définition des missions du musée que des enjeux de conservation préventive et d'ambiance.

\section{Le « nouveau musée » à l'épreuve des usages et des aléas}

\section{Mesures visant à fluidifier la circulation dans le musée}

Si l'accessibilité du musée inspire des retours plutôt positifs dans la presse, l'exiguïté unanimement reconnue des locaux fait craindre des problèmes de circulation. Le musée totalise 18000 visiteurs en une semaine, une fréquentation qui se stabilise les semaines suivantes, au point qu'il est même parfois impossible de circuler dans les salles de peintures. Si l'effet de nouveauté ne dure qu'un temps, de tels chiffres n'avaient manifestement pas été anticipés par le conservateur, peu habitué à une telle fréquentation avant le déménagement du musée, exception faite des jours suivant le vernissage des nouveaux accrochages. Dans ces conditions extrêmes, le parcours imposé en boucle entraîne des chassés-croisés et l'augmentation ponctuelle du débit de visiteurs fait craindre pour la préservation des collections exposées, notamment des sculptures. La préoccupation du conservateur est d'éviter autant que possible les chassés-croisés en faisant évoluer le trajet des visiteurs d'un parcours en boucle à un parcours linéaire, afin de mieux contrôler les entrées et sorties du musée, de favoriser l'évacuation en cas de mouvement de foule et de limiter le risque de détérioration des œuvres. La prudence et l'insistance du conservateur se heurtent à la résistance du président du Sénat, qui refuse obstinément qu'une porte de l'ancienne orangerie donnant sur son jardin privé soit utilisée comme porte de sortie pour le musée.

Une seconde mesure censée fluidifier la circulation dans le musée s'applique, sans négociation préalable, à l'une des principales catégories d'usagers du musée : les copistes. L'encombrement produit par l'accumulation des chevalets des copistes, notamment dans les salles les plus étroites, devient rapidement problématique. La décision unilatérale de limiter leur temps de travail à deux heures par jour soulève une protestation collective de ces artistes, déjà touchés par une mesure similaire dans l'ancien musée, en 1875. Ces derniers laissent entendre qu'il s'agit bien d'une erreur de programmation que l'on répercute sur leurs conditions de travail :

Veuillez remarquer, monsieur le conservateur, [...] que, si cette mesure rigoureuse est prise à cause de la petitesse des salles, il serait injuste de faire subir à ceux qui le déplorent le plus - et qui n'y sont pour rien - le vice de construction du nouveau musée [...] (Anonyme, 1886g)

Reprise et soutenue par le journal radical et satirique La Lanterne, la protestation des artistes copistes sort de la confidentialité ; en vain, ce quotidien exige d'une part que la réglementation sauvage imposée par Arago fasse l'objet d'un rapport avant d'être validée par l'administration des Beaux-Arts, et d'autre part, que le musée soit réservé une journée par semaine aux copistes pour leur permettre de travailler sans craindre de gêner les visiteurs. 


\section{Tentatives pour réguler le climat du musée} reprises sa hiérarchie sur l'urgence d'entreprendre des travaux pour réguler le climat du musée. L'administration des Beaux-Arts finit par autoriser l'architecte Charles Gondoin à prendre des mesures pour atténuer l'éclairage dans les salles (dépolissage des vitres des combles) et améliorer la ventilation dans les locaux (établissement de vasistas, de chatières et de prises d'air, installation de ventilateurs à hélice sur les combles). Toutefois, le manque - suspect - de transparence de l'administration des Beaux-Arts sur les raisons officielles de la fermeture du musée pour travaux en juillet 1886 se retourne contre elle, quand une explication plausible est donnée par trois copistes dans une lettre publiée une fois encore par La Lanterne :

Nous avons trouvé le musée fermé pour cause de travaux. Or, le Musée du Luxembourg vient d'être reconstruit entièrement, on est même resté sept mois à l'aménager, ce qui est plus que raisonnable. Il faut croire que le nouvel édifice, qui ressemble assez à une grange, a satisfait qui de droit puisque, à l'inauguration du monument?? on a décoré... le secrétaire de l'architecte !... un comble... sans mauvais jeu de mots. Mais alors, il est bien étrange que l'on soit déjà obligé de restaurer ou de réparer ou de compléter une œuvre remarquable! (Anonyme, 1886f)

Fustigeant au passage la tendance à l'autocongratulation de la maîtrise d'ouvrage et de la maitrise d'œuvre, les auteurs anonymes de la protestation sous-entendent l'incurie de la première tout en pointant l'incompétence et l'irresponsabilité de la seconde. C'est la question du climat intérieur du musée qui, pour ces copistes, a été manifestement sousestimée, soulignant l'absurdité du choix d'une orangerie pour y exposer des tableaux :

Il est vrai que l'on y étouffe ! $34^{\circ}$ dans la grande salle ; que les socles en faux marbre des statues qui se fendent de haut en bas; que les tableaux, brûlés par un soleil torride, s'écaillent et voient leurs plus belles nuances et leurs plus fins glacis se décomposer; que le public et les artistes sont exposés à une perpétuelle congestion cérébrale; et que les gardiens eux-mêmes sont malades; mais enfin tout cela est logique ; nous sommes dans l'ancienne Orangerie ; avec une candeur qui le rendrait digne des fleurs qu'abritait naguère son monument, l'architecte n'a rien changé à la disposition primitive de la serre. Serre elle était, serre elle est restée; malheureusement ni le public, ni les tableaux, ni les artistes n'ont rien de commun avec les orangers - exception faite pour quelques visiteuses bien entendu - et devant les maladresses faites, il faut après deux mois d'ouverture fermer le musée. (ibid.)

Deux ans après l'inauguration, le conservateur alerte sa tutelle sur la nécessité d'effectuer une réparation sur la toiture de la galerie de sculptures, cette fois en raison d'infiltrations d'eau pluviale. Après avoir procédé à une réparation ponctuelle de la galerie de sculptures en août 1888, par l'application de bandes de plomb sur les mastics et les joints des vitres, l'architecte obtient de l'administration des Beaux-Arts qu'elle finance également la réalisation du même travail sur les vitres des toitures de l'orangerie, afin de prévenir des risques similaires. 
Illustration 2 : [Jacques Gondoin, avec Georges Scellier de Gisors], coupe sur la galerie et la terrasse des sculptures du « nouveau musée ", s.d. [ca. 1884-1886 ?].

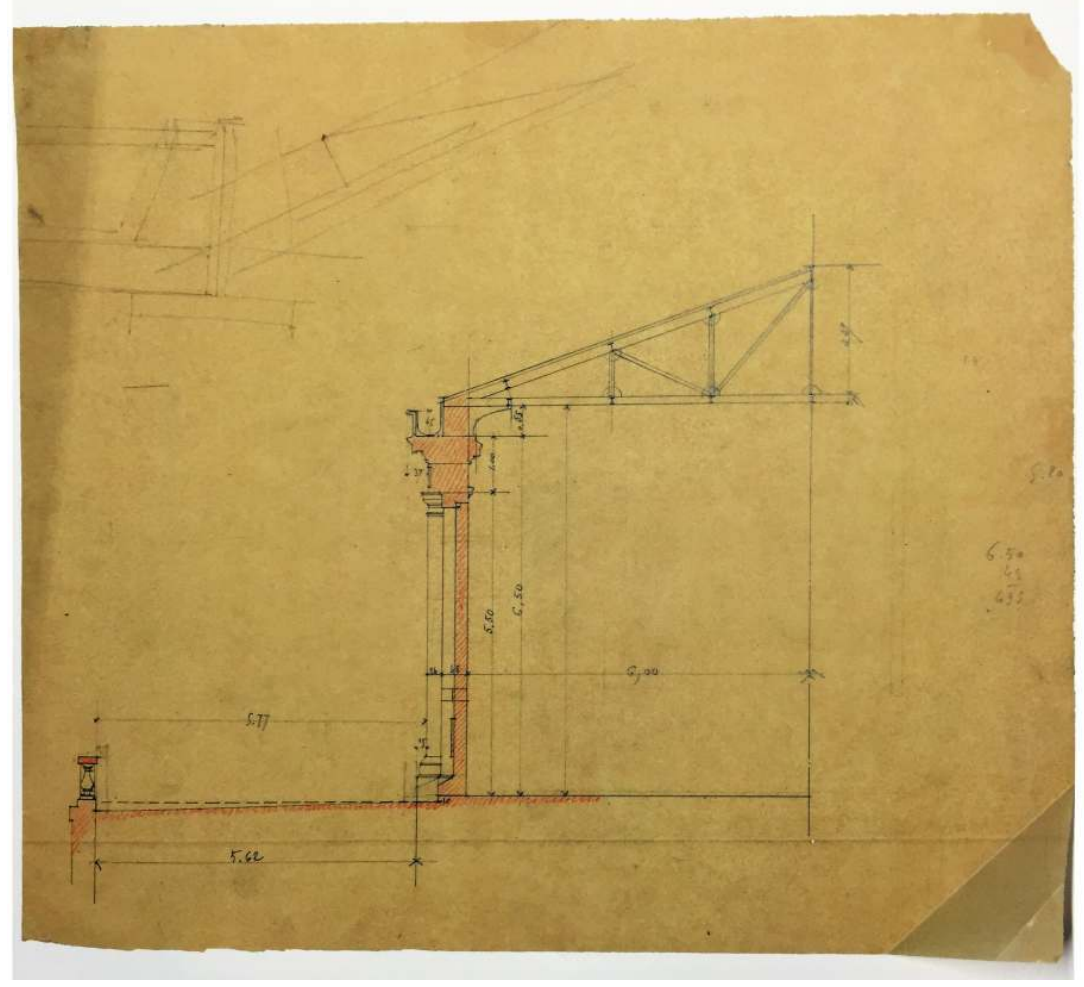

Source : Archives du Sénat, n 1338 (cote provisoire). Copyright : Archives du Sénat

\section{Un faisceau de négligences?}

\section{Un double compromis}

Cette série de mesures restrictives, correctives ou préventives prises dans les mois qui suivent l'inauguration tend à donner raison, rétrospectivement, aux journalistes les plus sceptiques ou les plus critiques, et doit nous inciter, en retour, à nous demander si ces problèmes d'ambiance ont été anticipés ou sous-estimés en amont de l'opération. Comment expliquer, en outre, le choix de l'orangerie pour y exposer des tableaux? Le croisement de correspondances administratives, de documents techniques produits par l'agence des architectes du palais du Luxembourg, et des comptes rendus du Conseil général des Bâtiments civils, ce comité d'architectes habilités à expertiser et amender les projets d'équipements publics, permet-il de vérifier l'hypothèse d'un faisceau de négligences dans les différentes phases du projet, de la faisabilité jusqu'au chantier?

Le choix de l'orangerie comme base du projet de relogement provisoire du Musée du Luxembourg apparaît comme la conséquence de deux compromis. Le premier de ces compromis résulte d'un double mouvement de résistance collective : résistance à un déracinement du musée de son quartier d'origine ; résistance au sacrifice d'une nouvelle partie du jardin du Luxembourg. En effet, l'hypothèse d'une reconstruction pérenne du Musée du Luxembourg à l'intérieur du jardin du Luxembourg fait resurgir un traumatisme collectif causé en 1866 par l'amputation, décrétée par le préfet de Paris, le 
baron Haussmann, des secteurs ouest et sud du jardin public pour en faire des terrains constructibles, en dépit d'une forte mobilisation populaire (Hopkins, 2001). L'un de ces secteurs, la pépinière, était devenu depuis la révolution de 1848 un lieu de promenade, dont l'ambiance bucolique était chantée par les poètes et les écrivains : « Pour les uns, elle devint un lieu de rêverie, pour les autres, une salle d'étude en plein air. Les étudiants y venaient, dans l'intervalle des cours, égayer de leurs vingt ans les nymphes mélancoliques ou les hamadryades délaissées » (Lacroix, 1867, p. 93). La polémique autour de la question du relogement du musée du Luxembourg, qui enfle notamment en 1883, agit alors comme un révélateur du recul qu'accuse la capitale française par rapport à sa rivale britannique, en termes de politique de valorisation et de développement des parcs et jardins publics : «Pendant que la ville de Londres agrandit encore ses immenses parcs intérieurs, qu'elle appelle très justement ses poumons, on pense à établir un massif de maçonnerie dans notre petit poumon de la rive gauche comme un tubercule dans la poitrine d'un phtisique » regrette Sabine Méa (Méa, 1883), qui ne cessera de prôner la sanctuarisation du jardin du Luxembourg. Réutiliser et agrandir l'orangerie permettait dès lors de limiter l'emprise des nouvelles constructions, et, par l'orientation de l'extension à cheval sur la limite entre la partie publique et la partie privée du jardin, de limiter son impact sur les plantations, à l'exception d'un parterre de fleurs, dont un expert du Conseil des Bâtiments civils regrette la disparition programmée :

[...] rien de plus gracieux que ce parterre, le tracé en est heureux et de plus, depuis bien des années, on s'est appliqué par le choix des plantations à faire de ce coin isolé du jardin un lieu de repos de soleil et de tranquillité, bien appréciés par le public intelligent qui vient y chercher un isolement qu'il ne saurait trouver ailleurs ${ }^{1}$

Le second compromis intervient au terme d'une longue phase de négociations entre le Sénat, gestionnaire du domaine du Luxembourg, et l'administration des Beaux-Arts, tutelle du musée. Les deux parties se mettent d'accord le 24 avril 1884 sur le principe de l'usufruit à titre gratuit, par lequel le Sénat octroierait un droit réel temporaire d'usage et de jouissance des bâtiments transformés de l'orangerie à la direction des Beaux-Arts, qui en serait alors le nu-propriétaire. En cas de reconstruction ultérieure et intégrale du musée, les bâtiments retourneraient au Sénat et pourraient remplir un autre usage. Cet accord implique, d'une part, que les nouveaux bâtiments doivent affecter un caractère générique, une monumentalité discrète, susceptible de convenir à n'importe quel type de programme, et d'autre part, que le Sénat est en position de force pour contrôler le contenu et les temporalités du projet architectural. 
Illustration 3 : [Jacques Gondoin, avec Georges Scellier de Gisors], plan du « nouveau musée » (état intermédiaire du projet d'aménagement et extension de l'orangerie Férou), s.d. [ca. 13 février 1884].

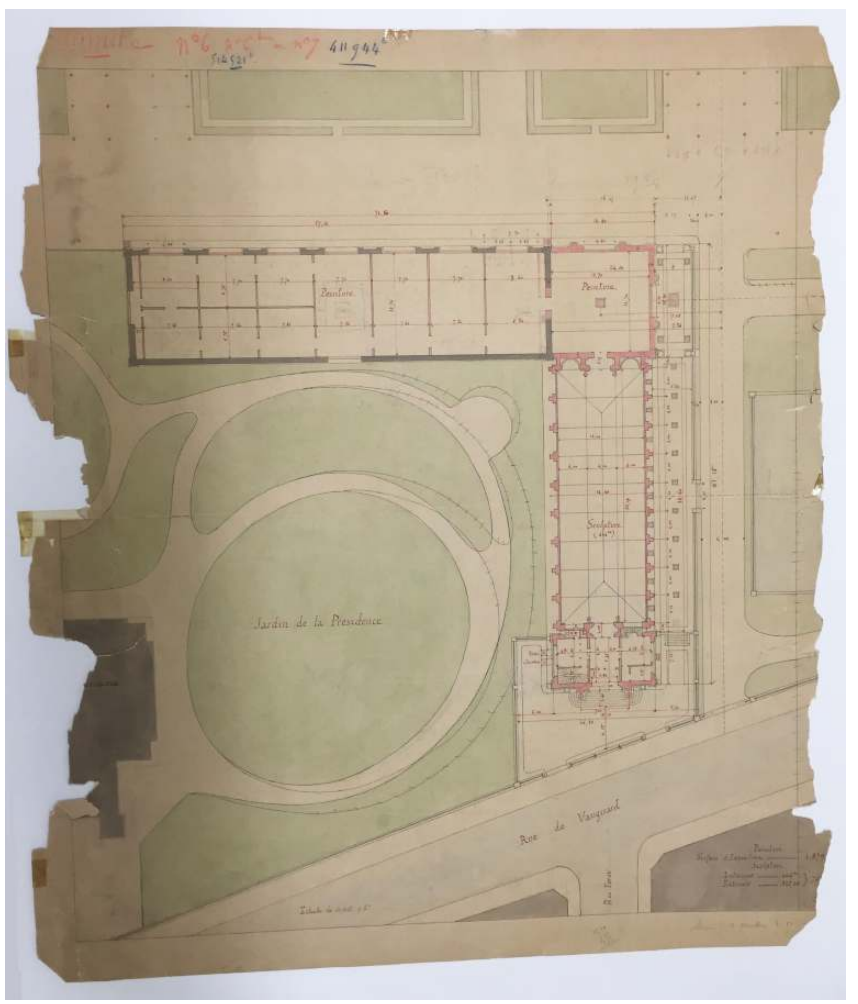

Source : Archives du Sénat, $n^{\circ} 2047$ (cote provisoire). Copyright : Archives du Sénat

\section{L'urgence contre la prudence}

L'examen des archives relatives aux différentes phases du projet, depuis les premières études de faisabilité en décembre 1879, jusqu'au chantier qui s'étale entre l'été 1884 et l'automne 1885, permet d'établir que la plupart des risques liés aux aléas et des problèmes d'ambiance évoqués au moment de l'inauguration sont, en réalité, connus non seulement des acteurs de l'opération mais aussi du grand public au plus tard en 1883. Sabine Méa figure alors parmi les journalistes les plus sceptiques sur la pertinence d'une réutilisation de l'orangerie pour en faire un musée de peintures :

Connaissez-vous l'Orangerie? Oui, sans doute; alors, vous comprenez tout ce que cette offre a de dérisoire. En vérité, une telle plaisanterie s'allie mal à la gravité habituelle du Sénat. Le bâtiment de l'Orangerie est insuffisant de toutes façons; il n'est pas assez vaste, il est mal éclairé, il n'a pas de fondations, et par conséquent ne peut être surélevé d'un étage. [...] Bref, l'installation des peintures à l'Orangerie est totalement impossible, et ce projet ne supporte pas l'examen. (Méa, 1883)

La certitude de l'absurdité d'un tel projet architectural, largement partagée dans la presse, s'impose pourtant tardivement au sein de la tutelle du musée. Début septembre 1884, alors que le chantier est à peine entamé, un délégué de l'administration des Beaux-Arts accompagné d'un architecte constatent, après une visite sur site, qu'« il serait préjudiciable pour les précieuses collections que renferme le musée de les transporter dans cet endroit» (Anonyme, 1884a). Bien que cette information soit abondamment relayée dans la presse, les travaux reprennent la semaine suivante. 
Hippolyte Gautier, journaliste au National, émet alors l'hypothèse que le Sénat aurait, en coulisses, exercé une pression sur l'administration des Beaux-Arts, pour que le chantier continue quoi qu'il arrive: «on nous apprend aujourd'hui que tout s'est arrangé : on passera par-dessus l'arrivée du délégué ; l'inspection était un peu tardive ; le mal était fait en partie ; autant qu'il s'achève " (Anonyme, 1884b). Cette pression pour que le chantier soit mené à son terme, le Sénat la justifie par l'impossibilité de retarder plus longtemps la mise à disposition des locaux occupés par le musée dans le palais du Luxembourg, pour faire face à l'accroissement des activités parlementaires. L'urgence de la mise en chantier a-t-elle pu avoir des conséquences sur le traitement des paramètres d'ambiance?

\section{Le traitement des paramètres d'ambiance dans le projet de restructuration-extension de l'orangerie du Luxembourg}

Les archives attestent que l'essentiel des paramètres d'ambiance évoqués au moment de l'inauguration le sont entre 1879 et 1884 . Pour ce qui est de la question de la lumière, Etienne Arago formule, dès les études de faisabilité (décembre 1879 et janvier 1880), le souhait que toutes les salles puissent recevoir un éclairage zénithal. Ses desiderata sont immédiatement pris en compte par l'architecte. Un comité de professionnels de la conservation, réuni en avril 1884, émet toutefois des réserves sur le principe d'une galerie de sculpture aux allures de serre ; l'architecte limite alors la présence du verre au comble et au plafond, solution retenue dans le projet final.

La question de la circulation du public est abordée à plusieurs moments du projet : dès 1879, le conservateur prône une circulation de plain-pied dans les salles; c'est également lui qui, au cours du chantier, en avril 1885, demande à l'architecte de supprimer les ouvertures faisant communiquer les trois premières salles de peinture et d'en percer de nouvelles dans l'axe de ces trois salles, arguant d'une part du risque d'obstruction du flux de visiteurs par la présence de copistes à proximité des portes, et d'autre part de l'amélioration des conditions de contemplation des œuvres. Le conservateur obtient gain de cause.

La question du climat intérieur du musée est partiellement traitée pendant la phase de programmation. Le Sénat anticipe notamment les froides journées d'hiver en prévoyant l'installation d'un chauffage par calorifères. Cependant, si l'architecte anticipe également le risque de surchauffe dans l'orangerie en établissant un second mur à l'intérieur du bâtiment, il sous-estime manifestement le risque de surchauffe dans la galerie de sculptures, ainsi que le risque d'humidité avant le lancement du chantier, alors même que la presse ne cesse d'insister sur ce point. L'architecte doit se rendre à l'évidence: l'humidité du sol sous et autour de l'orangerie l'oblige à repenser le dispositif d'évacuation des eaux pluviales qui se déversaient directement dans le sol et à créer un réseau de canalisations adapté, à établir une chape de béton sur toute la surface du bâtiment de l'orangerie, à consolider et étanchéifier la partie basse des murs de l'orangerie. En outre, les toitures du nouveau musée, conçues par l'entreprise SartoreMurat, spécialisée dans la construction de serres, sont équipées d'un système de tringles breveté, qui, « par son application à la jointure des verres, permet l'écoulement au dehors de la buée du vitrage, sans déperdition de chaleur» (Anonyme, 1885c). Cette solution, toutefois, s'avèrera donc insuffisante pour prévenir les infiltrations d'eau. à vouloir mener à son terme une opération compliquée, voire risquée pour les collections, 
suscite, entre 1883 et 1886, le scepticisme d'un grand nombre de journalistes ; l'un d'entre eux prophétise d'ailleurs que «l'expérience seule pourra nous édifier sur les qualités du nouveau musée ; espérons, comme dit un de nos confrères, qu'elle ne coûtera pas trop cher... aux tableaux » (Anonyme, 1884c).

\section{Conclusion}

41 L'analyse des récits de visite extraits d'un corpus restreint d'articles de presse a permis, dans un premier temps, de mettre en évidence le caractère contradictoire de la réception critique du nouveau siège du Musée du Luxembourg, au moment de son inauguration en 1886. L'observation des mesures restrictives, correctives et préventives, prises par la direction du musée dans les mois qui suivent l'ouverture au public du musée pour remédier à des problèmes d'ambiance, tendent à donner du crédit aux témoignages qui convergent vers une absence d'exemplarité architecturale et muséographique et incitent à interroger, en retour, les conditions dans lesquelles l'opération a été envisagée, conçue et réalisée. Il ressort de l'examen des archives et de la presse que l'orangerie du Luxembourg a été préférée à d'autres solutions plus pérennes, plus ambitieuses et plus adaptées, en raison du désengagement de l'administration des Beaux-Arts et d'une forte mobilisation populaire pour la sanctuarisation du jardin du Luxembourg. Mené à son terme malgré les avertissements de la presse, consciente des risques pour les collections, et les scrupules tardifs de la tutelle du Musée du Luxembourg, le projet architectural est pourtant modifié jusqu'à la fin du chantier par des imprévus ou des décisions liés aux enjeux d'ambiances (circulation, lumière, climat, en particulier). Ces modifications sont toutefois insuffisantes, pour remplir les exigences d'exemplarité formulées par le champ artistique. L'analyse des ambiances a donc pu nous servir de point de départ à la compréhension d'un moment de l'histoire culturelle française, où l'Etat se distingue par son incapacité à se donner les moyens de ses ambitions pour consolider son rayonnement artistique international.

\section{BIBLIOGRAPHIE}

Anonyme. 1883. Journal du Parlement. 04/04/1883.

Anonyme. 1884a. Le Matin. 04/09/1884.

Anonyme. 1884b. Journaux et revues. Gil Blas 17/10/1884.

Anonyme. 1884c. Menus faits. L'Univers illustré. 29/11/1884, p. 755.

Anonyme. 1885a. Le Musée du Luxembourg. Le Courrier de l'art. 30/10/1885. p. 528.

Anonyme. 1885b. Le nouveau Musée du Luxembourg. Le Temps. 18/09/1885.

Anonyme. 1885c. Le Figaro. 29/08/1885.

Anonyme. 1886a. La Vie à Paris. Le Temps. 04/04/1886. 
Anonyme. 1886b. Les on-dit. Le Rappel. 22/04/1886.

Anonyme. 1886c. Courrier de Paris. L'Univers illustré. 17/04/1886.

Anonyme. 1886d. Correspondances. Le Rappel. 15/04/1886.

Anonyme. 1886e. L'Illustration. 03/04/1886.

Anonyme. 1886f. Le Musée du Luxembourg. La Lanterne. 23/07/1886.

Anonyme. 1886g. Le Nouveau Luxembourg. La Lanterne. 30/11/1886.

Arago, Etienne. 1886. Introduction. Notice des peintures, sculptures et dessins de l'École moderne exposés dans les galeries du Musée national du Luxembourg. Paris: Imprimeries réunies.

Bastoen, Julien. 2015. L'Art contre l'Etat ? La trajectoire architecturale du Musée du Luxembourg dans la construction de l'illégitimité de l'action artistique publique 1848-1920. Thèse de doctorat en architecture. Marne-la-Vallée : Université Paris-Est.

Bertinet, Arnaud. 2015. Les Musées de Napoléon III. Une institution pour les arts (1849-1872). Paris : Mare et Martin.

Clément, Charles. 1886. Le nouveau Musée du Luxembourg. Journal des débats. 31/03/1886.

Daly, Marcel. Le nouveau Musée du Luxembourg. La Semaine des constructeurs. 22/05/1886.

Drozd, Céline. 2008a. Les ambiances dans la conception architecturale : une « histoire » de représentations. 1st International Congress on Ambiances. Grenoble 2008. Disponible sur : https:// halshs.archives-ouvertes.fr/halshs-00418340 (consulté le 28 octobre 2015).

Drozd, Céline. 2008b. Pour une approche sensible de l'architecture, le roman naturaliste. Equinoxes [en ligne]. Disponible sur : https://www.brown.edu/Research/Equinoxes/journal/ Issue \%2011/Eqx11_Drozd.html (consulté le 28 octobre 2015).

Drozd, Céline. 2011. Représentations langagières et iconographiques des ambiances architecturales : de l'intention d'ambiance à la perception sensible des usagers. Thèse de doctorat en architecture, Nantes : Ecole Centrale. Disponible sur https://tel.archives-ouvertes.fr/tel-00686046v2 (consulté le 28 octobre 2015).

Du Seigneur, Maurice. 1886. Le nouveau Musée du Luxembourg. La Construction moderne. 03/04/1886, p. 306-307.

Fournel, Victor. 1886. Les œuvres et les hommes. Le Correspondant. t. 143, $2^{\mathrm{e}}$ livraison, p. 336-359.

Georgel, Chantal. 1994. Montrer, éclairer, présenter. In : Georgel, Chantal (ed.). La Jeunesse des musées. Les Musées de France au XIX ${ }^{e}$ siècle. Paris : RMN. p. 188-206.

Grange, Sylvie \& Petit, M. 2007. 50 Lux et pas dans le noir ! Culture et recherche. $\mathrm{n}^{\circ}$ 113, (automne 2007), p. 22-23.

Hamon, Françoise. 2004. Musées européens au XIX ${ }^{\mathrm{e}}$ siècle. In : De Andia, Béatrice (dir.). Les Musées parisiens. Histoire, architecture et décor. Paris : Action artistique de la Ville de Paris. p. 58-69.

Hopkins, Richard S. 2011. Sauvons le Luxembourg : Urban Greenspace as Private Domain and Public Battleground, 1865-1867. Journal of urban studies. vol. 37, n 1, p. 43-58.

Jacques, Edmond. 1886. Le nouveau Musée du Luxembourg. L'Intransigeant. 01/04/1886.

Katow, Paul de. 1886. Le nouveau Musée du Luxembourg. Gil Blas. 01/04/1886. 
Lacambre, Geneviève. 2003. La constitution des collections nationales d'art moderne : le Musée du Luxembourg. In : Le Rôle de l'Etat dans la constitution des collections des musées de France et d'Europe. Paris : Direction des Musées de France. p. 91-113.

Lacroix, P. de. 1867. Histoire monumentale, pittoresque et anecdotique de la chartreuse de Paris, suivie d'une description nouvelle du Luxembourg et de ses jardins... Paris : Dumoulin.

Ladoué, Pierre. 1936. Musée du Luxembourg : le « Nouveau musée » de 1886. Bulletin des Musées de France. $n^{\circ} 10$ (12/1936), p. 184189.

Lorente, Jesús Pedro. 2009. Les musées d'art moderne ou contemporain : une exploration conceptuelle et historique. Paris : L'Harmattan.

Mantz, Paul. 1886. Le nouveau Musée du Luxembourg. Le Temps. 24/04/1886.

Méa, Sabine. 1883. Le Musée du Luxembourg. Le Rappel. 20/06/1883.

Méa, Sabine. 1886. Le Rappel. 06/04/1886.

Monnier, Gérard. 2006. Introduction. In : Monnier, Gérard (ed.). L’Architecture : la réception immédiate et la réception différée. Paris : Publications de la Sorbonne. p. 7-11.

Pomian, Krzysztof. Musées français, musées européens. In : Georgel, Chantal (ed.). La Jeunesse des musées. Les Musées de France au XIXe siècle. Paris : RMN. p. 351364.

Ponsonailhe, Charles. 1886. Le Rijks-Museum à Amsterdam et le nouveau Musée du Luxembourg. L'Artiste. t. 1, 1886, p. 341359.

Poulot, Dominique. 1983. La visite au musée au XIX siècle. Gazette des Beaux-Arts. 05/1983, p. 187-197.

Poulot, Dominique. 1994. Le musée et ses visiteurs. In : Georgel, Chantal (ed.). La Jeunesse des musées. Les Musées de France au XIX ${ }^{e}$ siècle. Paris : RMN. p. 332-351.

Roger. 1886. L'art et la curiosité. Le XIX'e siècle. 05/04/1886.

Roullin, Jean-François. 2002. La réception en architecture comme la réception en littérature? Cahiers thématiques. $\mathrm{n}^{\circ} 2$, p. 33-41.

Sherman, Daniel J. 1989. Worthy Monuments : Art Museums and the Politics of Culture in NineteenthCentury France. Cambridge (Mass.) : Harvard University Press.

Simonnot, Nathalie. 2011a. Ambiance et relation à l'œuvre - Perspective historique sur les musées français au XX ${ }^{\mathrm{e}}$ siècle. In : Augoyard, Jean-François (ed.). 2011. 1st International Congress on Ambiances. Grenoble 2008. Grenoble : A La Croisée. p. 460-464.

Simonnot, Nathalie. 2011b. Faire un musée en France entre 1955 et 1977. fabricA, travaux d'histoire culturelle et sociale de l'architecture et de ses territoires. $n^{\circ}$ 5, p. 112-129

Simonnot, Nathalie. 2012. La singularité des ambiances des musées de la Croissance en France (1945-1975). Figures de l'art. Revue d'études esthétiques. nº 21 (2012), p. 123-139.

\section{NOTES}

1. Louis-Jules André, rapport présenté au Conseil général des Bâtiments civils, 29 avril 1884, Archives nationales, $\mathrm{F}^{21} 6108$. 


\section{RÉSUMÉS}

Cet article propose de considérer les comptes rendus de visite au musée non pas du point de vue de l'expérience esthétique, ni même du point de vue de l'analyse sociologique du public des musées, mais comme une source pour l'histoire de l'architecture et la théorie de la réception (Roullin, 2002 ; Monnier, 2006). Par les enjeux d'ambiance qu'ils cristallisent (accessibilité, flux, confort, lumière, climat, etc.) et les contradictions qu'ils mettent en évidence, ces récits de visite peuvent être analysés comme des révélateurs de problématiques architecturales sous-estimées ou négligées dans le cadre du projet architectural. Le cas du nouveau siège du Musée du Luxembourg, inauguré en 1886 à Paris, est symptomatique de la manière dont le manque d'ambition de la politique de l'Etat français en faveur de l'art contemporain se traduit dans des choix architecturaux hasardeux, y compris et jusque dans le traitement partiel et approximatif des ambiances.

This paper considers museum visitor reviews neither in terms of aesthetic experience nor from a sociological point of view, but rather as a source of architectural history and the reception theory of architecture. As they deal with narratives on atmosphere (accessibility, flow, comfort, lightning, climate, etc.), visitor reviews published in general-interest newspapers or art and architecture journals address or reveal architectural issues that might have been underestimated and overlooked during the design process. The case of the new Luxembourg Museum building in Paris, inaugurated in 1886, is symptomatic of how the inconsistent policy of the French state regarding contemporary art affected the museum's design and atmosphere, as well as topics of preventive conservation.

\section{INDEX}

Keywords : atmosphere, reception, architecture, museum, contemporary art, art policy, user Mots-clés : ambiance, réception, architecture, musée, art contemporain, politique artistique, usager

\section{AUTEUR}

\section{JULIEN BASTOEN}

Julien Bastoen est historien et muséologue. Doctor europeus en architecture, il a consacré sa thèse à la trajectoire architecturale du Musée du Luxembourg entre 1848 et 1920 (Université Paris-Est Marne-la-Vallée, 2015). Il est ingénieur de recherche à l'IPRAUS / Ecole nationale supérieure d'architecture de Paris-Belleville (UMR Ausser n 3329 du CNRS). Contact : julien.bastoen@paris-belleville.archi.fr 